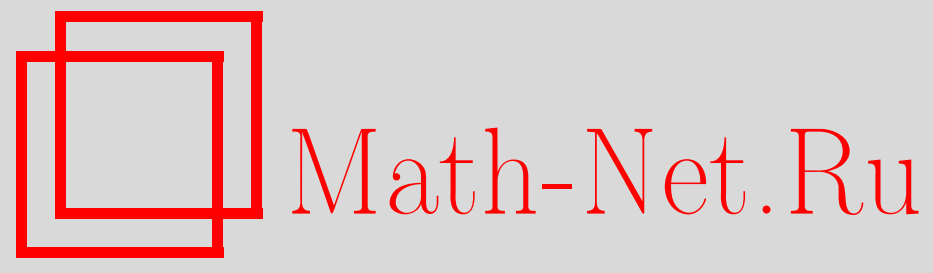

Ю. Н. Радаев, Правило множителей в ковариантных формулировках микрополярных теорий механики континуума, Вестн. Сам. гос. техн. ун-та. Сер. Физ.-мат. науки, 2018, номер 3, 504-517

DOI: https://doi.org/10.14498/vsgtu1635

Использование Общероссийского математического портала MathNet.Ru подразумевает, что вы прочитали и согласны с пользовательским соглашением

http://www. mathnet.ru/rus/agreement

Параметры загрузки:

IP: 52.205 .19 .152

26 апреля 2023 г., 15:57:29

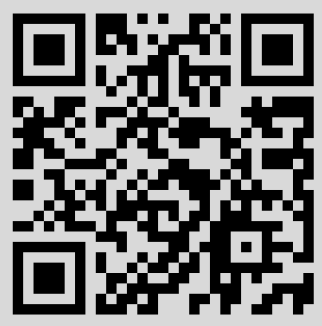


Вестн. Сам. гос. техн. ун-та. Сер. Физ.-мат. науки. 2018. Т. 22, № 3. С. $504-517$ ISSN: 2310-7081 (online), 1991-8615 (print) УДК 539.3

\title{
Правило множителей в ковариантных формулировках микрополярных теорий механики континуума
}

\section{Ю. Н. Радаев}

Институт проблем механики им А. Ю. Ишлинского РАН, Россия, 119526, Москва, пр-т Вернадского, 101, корп. 1.

\begin{abstract}
Аннотация
Рассматривается геометрически линейная модель микрополярного упругого тела (моментного континуума, континуума Коссера). Обсуждаются подходы к моделированию деформации таких континуумов. В качестве мер деформации выбираются: симметричный тензор малой деформации, вектор относительного микровращения и пространственный градиент вектора полного микровращения. Сформулированы принцип виртуальных перемещений и его обобщение, полученное с помощью метода неопределенных множителей Лагранжа, на основе которых выполнено построение микрополярной теории упругости. Важнейшей отличительной особенностью выступает отсутствие в вариационном уравнении вкладов работ внутренних силовых факторов, что позволяет придать принципу виртуальных перемещений весьма простую аналитическую форму. Подробно исследуется модель гемитропного микрополярного тела. Работа может рассматриваться как скрипт основных уравнений теории линейной микрополярной упругости, которые последовательно выводятся из принципа виртуальных перемещений с помощью правила множителей Лагранжа и в итоге образуют универсальную ковариантную формулировку всей теории.
\end{abstract}

Ключевые слова: микрополярный континуум, силовые напряжения, моментные напряжения, принцип виртуальных перемещений, множители Лагранжа, виртуальная работа, ковариантность.

Получение: 15 июля 2018 г. / Исправление: 23 августа 2018 г. / Принятие: 3 сентября 2018 г. / Публикация онлайн: 23 сентября 2018 г.

\section{Научная статья}

(2) (7) Контент публикуется на условиях лицензии Creative Commons Attribution 4.0 International (https://creativecommons.org/licenses/by/4.0/deed.ru)

\section{Образец для цитирования}

Р а д а е в Ю. Н. Правило множителей в ковариантных формулировках микрополярных теорий механики континуума // Вестн. Сам. гос. техн. ун-та. Сер. Физ.-мат. науки, 2018. T. 22 , № 3. C. 504-517. doi: 10.14498/vsgtu1635.

\section{Сведения об авторе}

Юрий Николаевич Радаев (1) http://orcid.org/0000-0002-0866-2151

доктор физико-математических наук, профессор; ведущий научный сотрудник; лаб. моделирования в механике деформируемого твердого тела; e-mail:radayev@ipmnet.ru, y.radayev@gmail.com 
Предварительные сведения и вводные замечания. Термины «работа» и «энергия» (фиксируя представление о том, что силы могут совершать «работу», механическая система может обладать «энергией») относятся к числу важнейших как в аналитической механике, так и в механике сплошных деформируемых сред [1-4]. Исторически понятие работы в исследованиях по механике систематически используется начиная с нового естествознания, т. е. более трех столетий, хотя в несколько завуалированной форме встречается уже в античную эпоху.

Согласно принципу возможных (виртуальных) перемещений (И. Бернулли, 1717 г.), малое и допускаемое связями перемещение системы из состояния равновесия может быть произведено без «затраты» работы. Значение принципа виртуальных перемещений заключается прежде всего в том, что при поиске уравнений равновесия механической системы, подчиненной геометрическим связям, нет никакой необходимости обладать какими-либо знаниями о том, как наложенные связи фактически реализуются, т.е. нет необходимости в определении сил реакций связей. Достаточно знать только все возможные виды перемещений, которые наложенные связи допускают, для системы, подчиненной связям. С прикладной точки зрения принцип виртуальных перемещений обладает одним неоспоримым преимуществом: он в одном вариационном уравнении соединяет совокупность всех условий механического равновесия.

В основу микрополярных теорий механики континуума может быть положен всего один принцип - принцип виртуальных перемещений. Вообще, принцип виртуальных перемещений занимает уникальное место в теории и механике сплошных деформируемых сред. В механике континуума принцип виртуальных перемещений обычно удобнее формулировать, используя только запас вариаций перемещений (а в случае микрополярной теории - еще и вариаций микроповоротов), соответствующих жестким перемещениям тела (и его жестким поворотам). Этим обеспечивается удивительная компактность и изящность математического представления этого принципа. Естественно, что правило множителей Лагранжа позволяет затем избавиться от такого рода ограничений на вариации перемещений и микровращений.

В настоящей работе принцип виртуальных перемещений в сочетании с правилом множителей Лагранжа применяется для вывода основных уравнений линейной теории микрополярной упругости. ${ }^{1}$ При этом в качестве мер деформации выбираются симметричный тензор деформации, вектор относительного вращения и пространственный градиент вектора полного вращения. Правило множителей является универсальным методом всего вариационного исчисления и широко используется даже за пределами границ его доказанной обоснованности. В случае микрополярной теории основная проблема - доказательство существования малых вариаций, не нарушающих наложенных дифференциальных ограничений, - сразу же получает свое решение: искомые вариации суть малые жесткие перемещения и повороты.

Работа может рассматриваться также и как скрипт основных уравнений линейной микрополярной упругости, которые последовательно выводятся из принципа виртуальных перемещений с помощью правила множите-

\footnotetext{
${ }^{1}$ Механике микрополярного континуума посвящено огромное количество работ. Мы укажем здесь только на одну из них - известную монографию В. Новацкого [5].
} 
лей Лагранжа. В большей степени это утверждение относится к модели гемитропного микрополярного тела, которое рассматривается в работе с необходимой полнотой. Определяющий гемитропное тело потенциал инвариантен по отношению ко всем собственно ортогональным преобразованиям, т.е. поворотам пространства, но его инвариантность нарушается по отношению к зеркальным отражениям и инверсиям пространства. Гемитропное (noncentrosymmetric, acentric, hemitropic or chiral) микрополярное упругое тело характеризуется сравнительно небольшим (в отличие от общей анизотропии) числом определяющих постоянных. Гемитропные материалы широко распространены в биологии, химии, оптике и многих других областях физики и в настоящее время интенсивно изучаются.

Изложение ведется исключительно в координатной форме и система координат в пространстве (оно считается трехмерным со стандартной евклидовой метрикой) предполагается криволинейной. Такой подход был выбран сознательно, поскольку в современной научной литературе невозможно найти представления уравнений микрополярной теории упругости в произвольных криволинейных координатах. Ясно, что как исходные, так и «окончательные» уравнения теории должны иметь объективную природу, хотя они и записываются с помощью частной пространственной координатной системы. Поэтому все уравнения необходимо будут иметь тензорную форму, т.е. ковариантную форму, пригодную для всех мыслимых координатных систем. Последнее обстоятельство вовсе не означает, что выбор системы координат в пространстве всегда безразличен. В прикладных вопросах благодаря удачному выбору координатной системы оказывается возможным учесть «симметрию» и существенно упростить выкладки, а также получить весьма простые и легко обозримые формы уравнений. Другой аспект затронутой проблемы - распознавание инвариантов, значения которых объективны и на самом деле не зависят от координатной системы (по поводу теории рациональных алгебраических инвариантов см. монографию [6]).

\section{1. Относительный микроповорот. Тензор чистой деформации.} Тензор изгиба-кручения. Классические модели континуума основываются на представлении о том, что континуум состоит из «обычных» материальных точек, положение которых в пространстве определяется их местом и которые совершают лишь поступательные движения вдоль (вообще говоря, криволинейных) траекторий. В этом смысле говорят о трех независимых трансляционных степенях свободы, которыми обладает каждая индивидуальная точка континуума. Широко известно обобщение данного представления, выполненное в 1909 г. Коссера [7]: каждая индивидуальная точка континуума наделяется тремя дополнительными вращательными степенями свободы и, следовательно, мыслится уже как элементарное свободное твердое тело, обладающее шестью степенями свободы. Это обобщение выглядит наиболее естественным среди очень большого разнообразия моделей современной механики континуума. Работа Коссера привлекла огромный интерес исследователей, правда, лишь полвека спустя.

Зафиксируем в пространстве некоторую координатную систему (вообще говоря, криволинейную). Обозначим через $x^{k}$ криволинейные координаты; здесь и в дальнейшем латинский индекс пробегает значения 1, 2, 3. Метрические свойства пространства полностью определяются метрическим тензором 
$g_{i j}$. Фундаментальный тензор $g^{i j}$ вводится с помощью уравнения $g^{i k} g_{k l}=\delta_{l}^{i}$. Квадрат инвариантного элемента длины вычисляется согласно

$$
d s^{2}=g_{i j} d x^{i} d x^{j}
$$

Квадратичная форма справа считается положительно определенной, откуда следует неравенство

$$
g=\operatorname{det}\left[g_{k l}\right]>0
$$

Знак детерминанта $g$ нельзя изменить никакими допустимыми преобразованиями координатной системы. Следовательно, корректно определяется квадратный радикал $\sqrt{g}$. Этот радикал используется для преобразования относительных (relative) тензоров в абсолютные (absolute) и, таким образом, позволяет не привлекать относительные тензоры для записи, в частности, уравнений микрополярной теории упругости.

В моментных теориях важную роль играют также символы перестановок $\epsilon^{i j k}, \epsilon_{i j k}$, которые с точки зрения закона преобразования являются относительными тензорами. Их можно легко превратить в абсолютные тензоры (так называемые $e$-тензоры) $e^{i j k}, e_{i j k}$. Сведения, касающиеся символов перестановок и $e$-тензоров, имеются во всех руководствах по многомерной геометрии и тензорному анализу (см., например, [8-10]). Приведем лишь один любопытный факт: символ перестановок $\epsilon_{i j k}$ является относительным ковариантным тензором веса -1 и одновременно - относительным контравариантным тензором веса +1 .

Поступательное перемещение индивидуальной точки может описываться трехмерным вектором перемещений (контравариантным абсолютным вектором) $u^{k}$. Поле поступательных перемещений порождает поле макроповоротов (macrorotation vector) - вихрь поля перемещений:

$$
\omega^{i}=\frac{1}{2} e^{i k l} \nabla_{k} u_{l}
$$

где

$$
\nabla_{i} u_{k}=\partial_{i} u_{k}-\Gamma_{i k}^{s} u_{s}
$$

есть ковариантная производная поля перемещений, $\Gamma_{i k}^{s}-$ символы Кристоффеля.

С индивидуальной точкой свяжем также микроповорот. Хорошо известно, что инфинитезимальный поворот в трехмерном пространстве представляется антисимметричным тензором второго ранга - тензор микроповорота (microrotation tensor) $\Omega_{i k}$ :

$$
\Omega_{i k}=\Omega_{[i k]}
$$

Здесь квадратные скобки обозначают операцию альтернирования по заключенным в них индексам. С антисимметричным тензором $\Omega_{i k}$ связывается вектор (micropolar microrotation vector) $\phi^{i}$ :

$$
\phi^{i}=\frac{1}{2} e^{i k l} \Omega_{k l} .
$$


Тензор $\Omega_{i k}$ может быть выражен через вектор $\phi^{i}$ согласно

$$
\Omega_{k l}=e_{k l j} \phi^{j}
$$

Таким образом, $u^{k}$ и $\phi^{i}$ - основные кинематические переменные микрополярной теории. Ясно, что картина деформации микрополярного континуума лучше всего описывается вектором относительного микровращения (relative microrotation vector) $\varphi^{i}$ :

$$
\varphi^{i}=\phi^{i}-\frac{1}{2} e^{i k l} \nabla_{k} u_{l}
$$

«Чистая» деформация при этом характеризуется симметричным тензором малых деформаций

$$
\epsilon_{(i j)}=\nabla_{(i} u_{j)}=\frac{1}{2}\left(\nabla_{i} u_{j}+\nabla_{j} u_{i}\right)
$$

где круглые скобки обозначают симметризацию по заключенным в них индексам. Тензор $\epsilon_{(i j)}$ - тензор малых деформаций, известный из классических теорий механики деформируемых тел.

Наконец, поле микроворотов $\phi^{i}$ порождает еще один тензор (тензор изгиба-кручения, wryness tensor), характеризующий деформацию изгиба-кручения:

$$
\kappa_{i \cdot}^{\cdot s}=\nabla_{i} \phi^{s}
$$

Определим также вектор $\kappa_{i}$, сопутствующий антисимметричной части тензора изгиба-кручения $\kappa_{i}^{\cdot s}$ :

$$
\kappa_{i}=\frac{1}{2} e_{i j s} \kappa^{[j s]}
$$

В подавляющем большинстве исследований по асимметричной упругости вводится «полный» асимметричный тензор деформации $\epsilon_{i j}$. Он конструируется из симметричного тензора деформации (1) и антисимметричного тензора

$$
\epsilon_{[i j]}=-e_{i j k} \varphi^{k}
$$

простым сложением тензоров (1) и (2):

$$
\epsilon_{i j}=\epsilon_{(i j)}+\epsilon_{[i j]} \text {. }
$$

Можно показать, что «полный» асимметричный тензор деформации вычисляется по формуле

$$
\epsilon_{i j}=\nabla_{i} u_{j}-e_{i j k} \phi^{k}
$$

2. Виртуальные перемещения и повороты. Виртуальная работа сил и моментов. Геометрические вариации полей перемещений и микроповоротов [11-13], не противоречащие наложенным связям, будем называть виртуальными перемещениями и микроповоротами и обозначать соответственно через $\delta u_{k}$ и $\delta \phi^{i}$. 
Вариации перемещений и поворотов при «жестком» движении должны удовлетворять следующим трем условиям:

$$
\begin{aligned}
& \nabla_{(i} \delta u_{k)}=0, \\
& \delta \varphi^{i}=\delta \phi^{i}-\frac{1}{2} e^{i k l} \nabla_{k} \delta u_{l}=0, \\
& \delta \kappa_{i \cdot}^{\cdot s}=\nabla_{i} \delta \phi^{s}=0 .
\end{aligned}
$$

Первое из них (3) выражает то известное обстоятельство, что жесткое движение не сопровождается деформацией (т.е. удлинениями и сдвигами), второе (4) - микроповорот неотличим от макроповорота, третье (5) - микроповороты не приводят к деформациям изгиба-кручения.

Силовыми факторами в моментных теориях выступают массовые силы $f^{k}$ и массовые пары $l_{k}$ (их объемные аналоги обозначим соответственно через $X^{k}=\rho f^{k}$ и $\left.Y_{k}=\rho l_{k}\right)$, а также поверхностные силы $t^{j}$ и моменты $m_{j}$.

Виртуальная работа, совершаемая всеми перечисленными выше силовыми факторами на виртуальных перемещениях $\delta u_{k}$ и микровращениях $\delta \phi^{i}$, без труда вычисляется так же, как это делается в аналитической механике:

$$
\delta A=\int\left[X^{j} \delta u_{j}+Y_{j} \delta \phi^{j}\right] d \tau+\oint_{\partial}\left[t^{j} \delta u_{j}+m_{j} \delta \phi^{j}\right] d S .
$$

Согласно принципу виртуальных перемещений, малое «жесткое» перемещение и «жесткий» поворот из состояния равновесия могут быть произведены без «затраты» работы, т.е. виртуальная работа обращается в нуль

$$
\delta A=0
$$

при наложенных на виртуальные перемещения и повороты дифференциальных ограничениях (3)-(5). Важнейшей отличительной особенностью здесь выступает отсутствие в вариационном уравнении (6) вкладов работ «внутренних» силовых факторов, что позволяет придать принципу виртуальных перемещений весьма простую аналитическую форму.

Таким образом, принцип виртуальных перемещений представляет собой вариационное уравнение (6) с наложенными на вариации дифференциальными связями (3)-(5). Поэтому дальнейшие рассуждения будут основываться на правиле множителей Лагранжа $[11,12]$. Вариационное уравнение (6) мы заменим на новое с неопределенными множителями, освободившись от связей (3)-(5). С этой целью исходя из структуры наложенных связей введем множители трех различных типов:

$-t^{(i k)}$ - первый (симметричный) тензорный множитель;

- $\tau_{j}$ - второй векторный множитель;

- $\mu_{. k}^{i}$ - третий тензорный множитель.

В результате вместо вариационного уравнения (6) получим новое вариационное уравнение со свободными вариациями $\delta u_{k}$ и $\delta \phi^{i}$ :

$$
\begin{aligned}
\int\left[X^{j} \delta u_{j}+Y_{j} \delta \phi^{j}-t^{(i k)} \nabla_{(i} \delta u_{k)}-2 \tau_{i}\left(\delta \phi^{i}\right.\right. & \left.\left.-\frac{1}{2} e^{i k l} \nabla_{k} \delta u_{l}\right)-\mu_{\cdot k}^{i \cdot} \nabla_{i} \delta \phi^{k}\right] d \tau+ \\
& +\oint_{\partial}\left[t^{j} \delta u_{j}+m_{j} \delta \phi^{j}\right] d S=0
\end{aligned}
$$


Выделяя в этом уравнении дивергентные слагаемые, применяя затем формулу Гаусса и вводя обозначение (второй антисимметричный тензорный множитель, являющийся вариантом второго множителя)

$$
t^{[i k]}=-e^{i k j} \tau_{j}
$$

приходим к следующему уравнению в вариациях $\left(n_{i}\right.$ - компоненты единичного вектора внешней нормали поверхности, ограничивающей рассматриваемый объем):

$$
\begin{aligned}
\int\left[X^{k}+\right. & \left.\nabla_{i} t^{(i k)}+\nabla_{i} t^{[i k]}\right] \delta u_{k} d \tau+\int\left[Y_{k}-2 \tau_{k}+\nabla_{i} \mu_{\cdot k}^{i \cdot}\right] \delta \phi^{k} d \tau+ \\
& +\oint_{\partial}\left[t^{k}-n_{i}\left(t^{(i k)}+t^{[i k]}\right)\right] \delta u_{k} d S+\oint_{\partial}\left[m_{k}-n_{i} \mu_{\cdot k}^{i \cdot}\right] \delta \phi^{k} d S=0 .
\end{aligned}
$$

3. Уравнения равновесия и динамики моментной теории. В силу произвольности вариаций $\delta u_{k}$ и $\delta \phi^{i}$ из уравнения (8) получаются дифференциальные уравнения равновесия, общие для всех моментных теорий:

$$
\begin{aligned}
& \nabla_{i} t^{i k}=-X^{k}, \\
& \nabla_{i} \mu_{\cdot k}^{i \cdot}-2 \tau_{k}=-Y_{k},
\end{aligned}
$$

где асимметричный неопределенный множитель вводится согласно

$$
t^{i k}=t^{(i k)}+t^{[i k]}
$$

Кроме этого, на поверхности получаются следующие соотношения:

$$
\begin{aligned}
& n_{i} t^{i k}=t^{k}, \\
& n_{i} \mu_{\cdot k}^{i \cdot}=m_{k} .
\end{aligned}
$$

Полученные результаты позволяют утверждать, что неопределенные множители $t^{(j k)}, \tau_{j}, \mu_{\cdot k}^{i}$ представляют собой реакции связей, налагаемых соответственно ограничениями, характеризующими «жесткие» перемещения и «жесткие» повороты:

$$
\begin{aligned}
& \epsilon_{(j k)}=0, \\
& \varphi^{j}=0, \\
& \kappa_{i \cdot}^{\cdot k}=0 .
\end{aligned}
$$

Ясно также, что с физической точки зрения тензоры $t^{j k}$ и $\mu_{\cdot k}^{i}$ суть тензор силовых напряжений (force stress tensor) и тензор моментных напряжений (couple stress tensor).

Уравнения равновесия (9) без затруднений обобщаются на динамический случай добавлением сил инерции $-\rho \ddot{u ̈}^{k},-\rho \mathfrak{I} \ddot{\phi}_{k}(\mathfrak{I}-$ момент микроинерции (spin torque constant)):

$$
\begin{aligned}
& \nabla_{i} t^{i k}=-\rho\left(f^{k}-\ddot{u}^{k}\right), \\
& \nabla_{i} \mu_{\cdot k}^{i \cdot}-2 \tau_{k}=-\rho\left(l_{k}-\mathfrak{I} \ddot{\phi}_{k}\right) .
\end{aligned}
$$


В дальнейшем изложении будут использоваться следующие антисимметричные тензоры и сопутствующие им векторы:

$$
\begin{aligned}
-\tau_{j} & =\frac{1}{2} e_{j i k} t^{[i k]}, \\
t^{[i k]} & =-e^{i k j} \tau_{j}, \\
+\mu^{i} & =\frac{1}{2} e^{i k s} \mu_{[k s]}, \\
\mu_{[i s]} & =+e_{i s j} \mu^{j} .
\end{aligned}
$$

4. Потенциал силовых и моментных напряжений. Гемитропное микрополярное тело. Принцип виртуальных перемещений, данный выше в форме с неопределенными множителями $(7),{ }^{2}$ преобразуется к виду

$$
\begin{aligned}
\int\left[t^{(i k)} \delta \nabla_{(i} u_{k)}+2 \tau_{i} \delta \varphi^{i}+\right. & \left.\mu_{\cdot k}^{i \cdot \delta} \delta \nabla_{i} \phi^{k}\right] d \tau= \\
& =\int\left[X^{j} \delta u_{j}+Y_{j} \delta \phi^{j}\right] d \tau+\oint_{\partial}\left[t^{j} \delta u_{j}+m_{j} \delta \phi^{j}\right] d S
\end{aligned}
$$

ИЛИ

$$
\begin{aligned}
\int\left[t^{(j k)} \delta \epsilon_{(j k)}+2 \tau_{j} \delta \varphi^{j}+\right. & \left.\mu_{\cdot k}^{j \cdot} \delta \kappa_{j \cdot}^{\cdot k}\right] d \tau= \\
& =\int\left[X^{j} \delta u_{j}+Y_{j} \delta \phi^{j}\right] d \tau+\oint_{\partial}\left[t^{j} \delta u_{j}+m_{j} \delta \phi^{j}\right] d S .
\end{aligned}
$$

Далее, принимая во внимание соотношения

$$
\begin{aligned}
& t^{j k} \delta \epsilon_{j k}=t^{(j k)} \delta \epsilon_{(j k)}+t^{[j k]} \delta \epsilon_{[j k]}=t^{(j k)} \delta \epsilon_{(j k)}+e^{j k l} e_{j k s} \tau_{l} \delta \varphi^{s}= \\
& =t^{(j k)} \delta \epsilon_{(j k)}+2 \delta_{s}^{l} \tau_{l} \delta \varphi^{s}=t^{(j k)} \delta \epsilon_{(j k)}+2 \tau_{s} \delta \varphi^{s}, \\
& 2 \mu^{j} \delta \kappa_{j}=\frac{1}{2} e^{j k l} \mu_{[k l]} e_{j p s} \delta \kappa^{[p s]}=\frac{1}{2} \mu_{[k l]} \delta \kappa^{[p s]}\left(\delta_{p}^{k} \delta_{s}^{l}-\delta_{p}^{l} \delta_{s}^{k}\right)=\mu_{[j k]} \delta \kappa^{[j k]},
\end{aligned}
$$

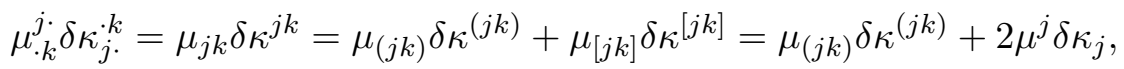

получаем альтернативные формулировки принципа виртуальной работы:

$$
\begin{aligned}
& \int\left[t^{(j k)} \delta \epsilon_{(j k)}+\mu_{(j k)} \delta \kappa^{(j k)}+2 \tau_{j} \delta \varphi^{j}+2 \mu^{j} \delta \kappa_{j}\right] d \tau= \\
&=\int\left[X^{j} \delta u_{j}+Y_{j} \delta \phi^{j}\right] d \tau+\oint_{\partial}\left[t^{j} \delta u_{j}+m_{j} \delta \phi^{j}\right] d S,
\end{aligned}
$$

$\int\left[t^{j k} \delta \epsilon_{j k}+\mu_{\cdot k}^{j \cdot} \delta \kappa_{j \cdot}^{\cdot k}\right] d \tau=\int\left[X^{j} \delta u_{j}+Y_{j} \delta \phi^{j}\right] d \tau+\oint_{\partial}\left[t^{j} \delta u_{j}+m_{j} \delta \phi^{j}\right] d S$.

\footnotetext{
${ }^{2} \mathrm{~B}$ текстах по механике сплошных сред и механике деформируемого твердого тела в этом случае говорят о «принципе виртуальной работы». Мы воспользуемся этим термином в дальнейших рассуждениях, хотя и будем отдавать себе отчет в том, что принцип виртуальной работы не является самостоятельным принципом, а представляет собой просто переформулировку принципа виртуальных перемещений с помощью метода множителей.
} 
Вторая из них (12) отчетливо демонстрирует сопряженность «полных» асимметричных силовых напряжений $t^{j k}$ и «полного» асимметричного тензора деформации $\epsilon_{j k}$, то же самое относится к моментным напряжениям $\mu_{\cdot k}^{j}$. и тензору изгиба-кручения $\kappa_{j}^{\cdot k}$. Она чаще всего используется в несимметричной теории упругости. Мы, тем не менее, рассмотрим первую из них (11). Содержательные следствия из (11) могут быть получены, если принять условие потенциальности симметричных силовых напряжений $t^{(j k)}$, симметричных моментных напряжений $\mu_{(i j)}$, а также векторных полей $\tau_{i}=, \mu^{i}$. Вводя потенциальную функцию $\mathscr{U}$ так, что

$$
\mathscr{U}=\mathscr{U}\left(\epsilon_{(i j)}, \kappa^{(i j)}, \varphi^{i}, \kappa_{i}\right),
$$

имеем

$$
\delta \mathscr{U}=t^{(i j)} \delta \epsilon_{(i j)}+\mu_{(i j)} \delta \kappa^{(i j)}+2 \tau_{i} \delta \varphi^{i}+2 \mu^{i} \delta \kappa_{i},
$$

и, кроме того, следующие определяющие уравнения:

$$
\begin{aligned}
t^{(i j)} & =\frac{\partial \mathscr{U}}{\partial \epsilon_{(i j)}}, \\
\mu_{(i j)} & =\frac{\partial \mathscr{U}}{\partial \kappa^{(i j)}}, \\
2 \tau_{i} & =\frac{\partial \mathscr{U}}{\partial \varphi^{i}}, \\
2 \mu^{i} & =\frac{\partial \mathscr{U}}{\partial \kappa_{i}} .
\end{aligned}
$$

Эти определяющие уравнения можно обратить, если воспользоваться преобразованием Лежандра.

Заметим, что в силу (13) принцип виртуальной работы (11) приобретает вид

$$
\int \delta \mathscr{U} d \tau=\int\left[X^{j} \delta u_{j}+Y_{j} \delta \phi^{j}\right] d \tau+\oint_{\partial}\left[t^{j} \delta u_{j}+m_{j} \delta \phi^{j}\right] d S .
$$

В том случае, когда потенциал $\mathscr{U}$ инвариантен относительно гемитропной группы, он представляется в форме

$$
\begin{aligned}
& \mathscr{U}=\underset{1}{A} g^{i s} g^{l m} \epsilon_{(i s)} \epsilon_{(l m)}+\underset{2}{A} g_{i s} g_{l m} \kappa^{(i s)} \kappa^{(l m)}+\underset{3}{A} g^{i s} g^{l m} \epsilon_{(i l)} \epsilon_{(s m)}+ \\
& +\underset{4}{A} g_{i s} g_{l m} \kappa^{(i l)} \kappa^{(s m)}+\underset{5}{A} g_{i s} \varphi^{i} \varphi^{s}+\underset{6}{A} g^{i s} \kappa_{i} \kappa_{s}+ \\
& +\underset{7}{A} g^{i s} g_{l m} \epsilon_{(i s)} \kappa^{(l m)}+\underset{8}{A} \epsilon_{(i s)} \kappa^{(i s)}+\underset{9}{A} \kappa_{i} \varphi^{i},
\end{aligned}
$$

где $\underset{i}{A}(i=1, \ldots, 9)$ - определяющие постоянные (коэффициенты жесткости). Тогда определяющие уравнения получаются в виде

$$
\begin{aligned}
& t^{(i s)}=\underset{1}{2 A} g^{i s} g^{l m} \epsilon_{(l m)}+2 \underset{3}{A g^{i l}} g^{s m} \epsilon_{(l m)}+\underset{7}{A} g^{i s} g_{l m} \kappa^{(l m)}+\underset{8}{A} \kappa^{(i s)}, \\
& \mu_{(i s)}=\underset{2}{2 A} g_{i s} g_{l m} \kappa^{(l m)}+\underset{4}{2 A} g_{i l} g_{s m} \kappa^{(l m)}+\underset{7}{A} g_{i s} g^{l m} \epsilon_{(l m)}+\underset{8}{A} \epsilon_{(i s)}, \\
& 2 \tau_{i}=\underset{5}{A g_{i s}} \varphi^{s}+\underset{9}{A} \kappa_{i}, \\
& 2 \mu^{i}=\underset{6}{2 A} g^{i s} \kappa_{s}+\underset{9}{A} \varphi^{i} .
\end{aligned}
$$


Вместо девяти коэффициентов жесткости $\underset{i}{A}$, появляющихся в выражении для упругого потенциала (14), удобнее ввести другие определяющие постоянные:

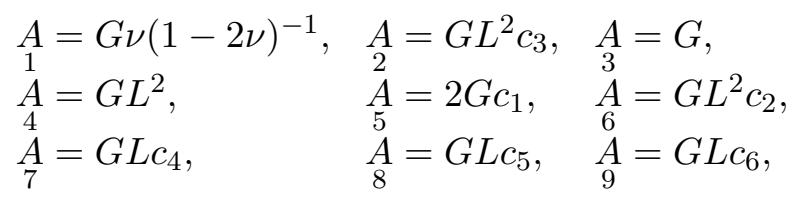

с тем чтобы в итоге пришлось иметь дело с двумя размерными и семью безразмерными постоянными:

- $G$ - модуль сдвига (имеет размерность силовых напряжений);

- $\nu$ - коэффициент Пуассона (не имеет физической размерности);

- $L$ - характерная длина микрополярной теории;

- $c_{1}, c_{2}, c_{3}, c_{4}, c_{5}, c_{6}$ - не имеющие физической размерности постоянные.

В результате вместо (15) приходим к определяющим уравнениям гемитропной микрополярной среды:

$$
\begin{aligned}
& t^{(i s)}=2 G\left(\nu(1-2 \nu)^{-1} g^{i s} g^{l m}+g^{i l} g^{s m}\right) \epsilon_{(l m)}+G L\left(c_{4} g^{i s} g_{l m} \kappa^{(l m)}+c_{5} \kappa^{(i s)}\right), \\
& \mu_{(i s)}=2 G L^{2}\left(c_{3} g_{i s} g_{l m}+g_{i l} g_{s m}\right) \kappa^{(l m)}+G L\left(c_{4} g_{i s} g^{l m} \epsilon_{(l m)}+c_{5} \epsilon_{(i s)}\right), \\
& \tau_{i}=2 G c_{1} g_{i s} \varphi^{s}+\frac{1}{2} G L c_{6} \kappa_{i}, \\
& \mu^{i}=G L^{2} c_{2} g^{i s} \kappa_{s}+\frac{1}{2} G L c_{6} \varphi^{i},
\end{aligned}
$$

или для «полных» силовых и моментных напряжений:

$$
\begin{gathered}
t_{i s}=G\left[\left(1+c_{1}\right) \nabla_{i} u_{s}+\left(1-c_{1}\right) \nabla_{s} u_{i}+2 \nu(1-2 \nu)^{-1} g_{i s} \nabla_{k} u^{k}-\right. \\
\left.-2 c_{1} e_{i s l} \phi^{l}+L c_{4} g_{i s} \nabla_{l} \phi^{l}+L c_{5} \nabla_{(i} \phi_{s}-\frac{1}{2} L c_{6} \nabla_{[i} \phi_{s]}\right], \\
\mu_{i s}=G L^{2}\left[\left(1+c_{2}\right) \nabla_{i} \phi_{s}+\left(1-c_{2}\right) \nabla_{s} \phi_{i}+2 c_{3} g_{i s} \nabla_{l} \phi^{l}+\right. \\
\left.+L^{-1}\left(c_{4} g_{i s} \nabla_{l} u^{l}+c_{5} \nabla_{(i} u_{s)}-\frac{1}{2} c_{6} \nabla_{[i} u_{s]}+\frac{1}{2} c_{6} e_{i s l} \phi^{l}\right)\right] .
\end{gathered}
$$

Динамические уравнения для микрополярного гемитропного тела получаются подстановкой выражений для «полных» силовых и моментных напряжений (16) в (10) и находятся в следующем виде:

$$
\begin{array}{r}
G\left[\left(1+c_{1}\right) \nabla^{s} \nabla_{s} u^{i}+\left(1-c_{1}+2 \nu(1-2 \nu)^{-1}\right) \nabla^{i} \nabla_{k} u^{k}+2 c_{1} e^{i k l} \nabla_{k} \phi_{l}+\right. \\
\left.+L c_{4}^{\prime} \nabla^{i} \nabla_{k} \phi^{k}+L c_{5}^{\prime} \nabla^{k} \nabla_{k} \phi^{i}\right]=-\rho\left(f^{i}-\ddot{u}^{i}\right), \\
G L^{2}\left[\left(1+c_{2}\right) \nabla^{s} \nabla_{s} \phi_{i}+\left(1-c_{2}+2 c_{3}\right) \nabla_{i} \nabla_{k} \phi^{k}+L^{-1} c_{4}^{\prime} \nabla_{i} \nabla^{k} u_{k}+\right. \\
\left.+L^{-1} c_{5}^{\prime} \nabla^{k} \nabla_{k} u_{i}+L^{-1} c_{6}^{\prime} e_{i s l} \nabla^{s} \phi^{l}\right]-2 G c_{1}\left(2 \phi_{i}-e_{i k l} g^{k s} \nabla_{s} u^{l}\right)= \\
=-\rho\left(l_{i}-\mathfrak{I} \ddot{\phi}_{i}\right),
\end{array}
$$

где введены обозначения

$$
c_{4}^{\prime}=c_{4}+\frac{1}{2} c_{5}+\frac{1}{4} c_{6}, \quad c_{5}^{\prime}=\frac{1}{2} c_{5}-\frac{1}{4} c_{6}, \quad c_{6}^{\prime}=-c_{6} .
$$


Уравнение (17) пригодно для любой криволинейной координатной системы в пространстве; иногда приходится оперировать с векторной формой динамических уравнений, которая приводится ниже:

$$
\begin{aligned}
& G\left[\left(1+c_{1}\right) \boldsymbol{\nabla} \cdot \boldsymbol{\nabla} \mathbf{u}+\left(1-c_{1}+2 \nu(1-2 \nu)^{-1}\right) \nabla \nabla \cdot \mathbf{u}+\right. \\
& \left.+2 c_{1} \nabla \times \boldsymbol{\phi}+L c_{4}^{\prime} \nabla \nabla \cdot \boldsymbol{\phi}+L c_{5}^{\prime} \boldsymbol{\nabla} \cdot \boldsymbol{\nabla} \boldsymbol{\phi}\right]=-\rho(\mathbf{f}-\ddot{\mathbf{u}}), \\
& G L^{2}\left[\left(1+c_{2}\right) \boldsymbol{\nabla} \cdot \boldsymbol{\nabla} \boldsymbol{\phi}+\left(1-c_{2}+2 c_{3}\right) \nabla \boldsymbol{\nabla} \cdot \boldsymbol{\phi}+L^{-1} c_{4}^{\prime} \nabla \nabla \cdot \mathbf{u}+\right. \\
& \left.+L^{-1} c_{5}^{\prime} \nabla \cdot \nabla \mathbf{u}+L^{-1} c_{6}^{\prime} \nabla \times \boldsymbol{\phi}\right]-2 G c_{1}(2 \phi-\nabla \times \mathbf{u})=-\rho(\mathbf{l}-\Im \ddot{\boldsymbol{\phi}}) .
\end{aligned}
$$

Это уравнение, записанное в терминах вектора перемещений $\mathbf{u}$ и вектора полных микровращений $\phi$, служит основой для исследования сильных и слабых разрывов в микрополярной гемитропной среде, а также волновых процессов, которые в рассматриваемом случае характеризуются одновременным распространением лево- и правоориентированных волн перемещений и микровращений.

\section{Заключение.}

1. Деформация микрополярного континуума описывается с помощью трех линейных мер: симметричного тензора малой деформации, вектора относительного микровращения и пространственного градиента вектора полного микровращения (тензора изгиба-кручения).

2. Исходя из принципа виртуальных перемещений с наложенными дифференциальными связями и правила множителей Лагранжа получены статические и динамические уравнения теории микрополярного тела. Принцип обеспечивает универсальную ковариантную формулировку всей теории.

3. Принцип виртуальных перемещений обобщается с помощью правила множителей и формулируется принцип виртуальной работы для микрополярных теорий.

4. Построен определяющий гемитропное упругое тело потенциал, инвариантный по отношению ко всем собственно ортогональным преобразованиям, т.е. поворотам пространства. Его инвариантность нарушается по отношению к преобразованиям зеркального отражения и инверсий трехмерного пространства.

5. Введены определяющие постоянные гемитропного упругого тела (две размерных и семь физически безразмерных).

6. Получены динамические уравнения (в терминах вектора перемещений и вектора полных микровращений) с целью моделирования волновых процессов в микрополярных гемитропных средах.

Конкурирующие интересы. Конкурирующих интересов не имею.

Авторская ответственность. Я несу полную ответственность за предоставление окончательной версии рукописи в печать. Окончательная версия рукописи мною одобрена.

Финансирование. Работа выполнена по теме государственного задания (№ государственной регистрации АААА-A17-117021310381-8) и при частичной финансовой поддержке Российского фонда фундаментальных исследований (проект № 18-0100844_а«Моделирование термомеханических процессов в сложных средах с помощью принципа термомеханической ортогональности»). 


\section{Библиографический список}

1. Планк М. Ведение в теоретическую физику. Часть первая. Общая механика. М., Л.: Гостехтеоретиздат, 1932. 200 с.

2. Лурье А. И. Аналитическая механика. М.: Физматгиз, 1961. 824 с.

3. Арнольд В. И. Математические методы классической механики. М.: Наука, 1974. $432 \mathrm{c}$.

4. Седов Л. И. Введение в механику сплошных сред. М.: Физматгиз, 1962. 284 с.

5. Nowacki W. Teoria sprężystości. Państwowe Wydawnictwo Naukowe: Warszawa, 1970. 769 pp. (In Polish); Новацкий В. Теория упругости. М.: Мир, 1975. 872 с.

6. Гуревич Г. Б. Основы теории алгебраических инвариантов. М., Л.: Гостехтеоретиздат, 1948. 408 c.

7. Cosserat E., Cosserat F. Théorie des corps déformables. Paris: A. Hermann et Fils, 1909, http://resolver.library. cornell.edu/math/1878913.

8. Розенфельд Б. А. Многомерные пространства. М.: Наука, 1966. 648 с.

9. Рашевский П. К. Риманова геометрия и тензорный анализ. М.: Наука, 1964. 664 с.

10. Сокольников И. С. Тензорный анализ. Теория и применения в геометрии и в механике сплошных сред. М.: Наука, 1971. 376 с.

11. Гюнтер Н. М. Курс вариационного исчисления. М., Л.: Гостехтеоретиздат, 1941. 308 с.

12. Гельфанд И. М., Фомин С. В. Вариачионное исчисление. М.: Физматгиз, 1961. 228 с.

13. Ковалев В. А., Радаев Ю. Н. Элементы теории поля: вариационные симметрии и геометрические инварианты. М.: Физматлит, 2009. 156 с. 


\title{
MSC: 74A60, 74F05
}

\section{The Lagrange multipliers method in covariant formulations of micropolar continuum mechanics theories}

\section{Y. N. Radayev}

Ishlinsky Institute for Problems in Mechanics of the Russian Academy of Sciences,

101-1, pr. Vernadskogo, Moscow, 119526, Russian Federation.

\begin{abstract}
Linear model of micropolar elastic continuum (known also as the Cosserat continuum) is considered. Kinematics and strain measures are discussed. The symmetric small strains tensor, relative microrotation vector and spatial gradient of the total microrotation vector (the wryness tensor) are then employed for a covariant formulation of the micropolar theory. By means of the principle of virtual displacements much simplified by the lack of internal forces and couples contributions to the virtual work and the Lagrange multipliers method the micropolar theory of elasticity is developed. Hemitropic micropolar continuum model is investigated in further details. The paper is to be considered as a universal covariant script of equations of the linear micropolar theory of elasticity derived from the virtual displacements principle.
\end{abstract}

Keywords: micropolar continuum, force stress, couple stress, virtual displacements principle, Lagrange multipliers, virtual work, covariant formulation.

Received: $15^{\text {th }}$ July, 2018 / Revised: $23^{\text {rd }}$ August, $2018 /$ Accepted: $3^{\text {rd }}$ September, 2018 / First online: $23^{\text {rd }}$ September, 2018

Competing interests. I declare that I have no competing interests.

Author's Responsibilities. I take full responsibility for submitting the final manuscript in print. I approved the final version of the manuscript.

Funding. This study was in part financially supported by the Federal Agency for Scientific Organizations (State Registration Number AAAA-A17-117021310381-8) and by the Russian Foundation for Basic Research (project no. 18-01-00844_a).

\section{Research Article}

( ()(1) The content is published under the terms of the Creative Commons Attribution 4.0 International License (http://creativecommons.org/licenses/by/4.0/)

Please cite this article in press as:

Radayev Y. N. The Lagrange multipliers method in covariant formulations of micropolar continuum mechanics theories, Vestn. Samar. Gos. Tekhn. Univ., Ser. Fiz.-Mat. Nauki [J. Samara State Tech. Univ., Ser. Phys. Math. Sci.], 2018, vol. 22, no. 3, pp. 504-517. doi: 10.14498/vsgtu1635 (In Russian).

Author's Details:

Yuri N. Radayev (10) http://orcid.org/0000-0002-0866-2151

Dr. Phys. \& Math. Sci., PhD, MSc, Professor; Leading Researcher; Lab. of Modeling in Solid Mechanics; e-mail: radayev@ipmnet.ru, y.radayev@gmail.com 


\section{References}

1. Planck M. Einführung in die theoretische Physik. Bd. I: Einführung in die allgemeine Mechanik. Leipzig, S. Hirzel, 1928, viii+226 pp. (In German)

2. Lurie A. I. Analytical mechanics, Foundations of Engineering Mechanics. Berlin, Springer, 2002, 864 pp.

3. Arnold V. I. Mathematical methods of classical mechanics, Graduate Texts in Mathematics, vol. 60. New York, Heidelberg, Berlin, Springer-Verlag, 1978, x+462 pp.

4. Sedov L. I. Introduction to the mechanics of a continuous medium. Addison-Wesley Publ., 1965, xvi $+270 \mathrm{pp}$.

5. Nowacki W. Teoria sprężystości. Państwowe Wydawnictwo Naukowe, Warszawa, 1970, 769 pp. (In Polish)

6. Gurevich G. B. Foundations of the theory of algebraic invariants. Groningen, The Netherlands, P. Noordhoff Ltd., 1964, viii +429 pp.

7. Cosserat E., Cosserat F. Théorie des corps déformables. Paris, A. Hermann et Fils, 1909, http://resolver.library. cornell.edu/math/1878913.

8. Rosenfeld B. A. Multidimensional Spaces, In: A History of Non-Euclidean Geometry, Studies in the History of Mathematics and Physical Sciences, 12. New York, Springer, 1988. doi : 10. 1007/978-1-4419-8680-1_7.

9. Rashevskii P. K. Rimanova geometriia i tenzornyi analiz [Riemannian geometry and tensor analysis]. Moscow, Nauka, 1964, 664 pp. (In Russian)

10. Sokolnikoff I. S. Tensor analysis. Theory and applications to geometry and mechanics of continua, Applied Mathematics Series. New York, London, Sydney, John Wiley and Sons, Inc., 1964, xii $+361 \mathrm{pp}$.

11. Gyunther N. M. Kurs variatsionnogo ischisleniia [A course of the calculus of variations]. Moscow, Leningrad, Gostekhteoretizdat, 1941, 308 pp. (In Russian)

12. Gelfand I. M., Fomin S. V. Calculus of variations. Englewood Cliffs, N.J., Prentice-Hall, Inc, 1965, vii $+232 \mathrm{pp}$.

13. Kovalev V. A., Radayev Yu. N. Elementy teorii polia: variatsionnye simmetrii $i$ geometricheskie invarianty [Elements of the Field Theory: Variational Symmetries and Geometric Invariants]. Moscow, Fizmatlit, 2009, 156 pp. (In Russian) 